\title{
Phase Transitions in Quasi-One-Dimensional System with Unconventional Superconductivity
}

\author{
Andrzej Ptok ${ }^{1,2}$ (1) Agnieszka Cichy ${ }^{3,4}$ - Karen Rodríguez ${ }^{5,6}$. \\ Konrad Jerzy Kapcia ${ }^{1,7}$ (1)
}

Received: 19 September 2017 / Accepted: 21 September 2017 / Published online: 3 October 2017

(C) The Author(s) 2017. This article is an open access publication

\begin{abstract}
The paper is devoted to a study of superconducting properties of population-imbalanced fermionic mixtures in quasi-one-dimensional optical lattices. The system can be effectively described by the attractive Hubbard model with the Zeeman magnetic field term. We investigated the ground-state phase diagram of the model as a function of the chemical potential and the magnetic field. The ground
\end{abstract}

Konrad Jerzy Kapcia

konrad.kapcia@ifpan.edu.pl

Andrzej Ptok

aptok@mmj.pl

Agnieszka Cichy

agnieszkakujawa2311@gmail.com

Karen Rodríguez

karem.c.rodriguez@correounivalle.edu.co

1 Institute of Nuclear Physics, Polish Academy of Sciences, ul. E. Radzikowskiego 152, PL-31342 Kraków, Poland

2 Institute of Physics, Maria Curie-Skłodowska University, Plac M. Skłodowskiej-Curie 1, PL-20031 Lublin, Poland

3 Solid State Theory Division, Faculty of Physics, Adam Mickiewicz University in Poznań, ul. Umultowska 85, PL-61614 Poznań, Poland

4 Institut für Physik, Johannes Gutenberg-Universität Mainz, Staudingerweg 9, 55099 Mainz, Germany

5 Departamento de Física, Universidad del Valle, A.A. 25360, Cali, Colombia

6 Centre for Bioinformatics and Photonics - CiBioFi, Calle 13 No. 100-00, Edificio 320 No. 1069, Cali, Colombia

7 Institute of Physics, Polish Academy of Sciences, Aleja Lotników 32/46, PL-02668 Warsaw, Poland state of the system exhibits the conventional BCS-type superconductivity as well as the unconventional so-called Fulde-Ferrell-Larkin-Ovchinnikov state, in which the total momentum of Cooper pairs is non-zero. We determine the orders of transitions as well as the behavior of order parameters with a change of the model parameters.

Keywords Attractive Hubbard model · FFLO superconductivity · Ground state · Phase diagram . Phase transition

\section{Introduction}

The unconventional superconductivity is still very fascinating but unsolved problem. It involves such issues as superconductivity with extremely short coherence length [1-4], the BCS-BEC crossover [4-6], unconventional pairings (particularly those with non-zero total momentum of Cooper pairs, $\boldsymbol{Q} \neq 0$, e.g., the so-called Fulde-FerrellLarkin-Ovchinnikov (FFLO) state [7-9]). The phenomenon of the FFLO superconductivity can be realized possibly in many physical systems: (i) in condensed matter physics, e.g., iron-based superconductors [10-14], heavy-fermion compounds [14-20], and organic conductors [21-23], as well as (ii) in ultra cold atomic gases on optical lattices [2434]. In the latter group of systems, the interaction parameters can be changed in a wider range [35-39]. Thus, it makes that group better candidate to experimentally investigate the model Hamiltonians.

Motivated by the experimental feasibility of such systems with ultracold gases loaded on a quasi-one-dimensional lattice, we studied the unconventional superfluid phases of the attractive Hubbard model, in the presence of an external magnetic field [40]. In that paper, we have shown that the 
system evolves from the BCS-type superconducting state (at small field) to the FFLO phase (for sufficiently large field). In an extremal case, the momentum of Cooper pairs can lie on the vertex of the first Brillouin zone and the so-called $\eta$ phase emerges. In this work, we present and discuss in details the dependence (as a function of chemical potential and magnetic field) of the following quantities: (i) an amplitude of superconducting order parameter, (ii) a total momentum of Cooper pairs, and (iii) the particle concentration.

The rest of the paper is organized as follows. In Section 2, the investigated model is presented and the method of its solution is briefly discussed. Section 3 is devoted to a discussion of the ground-state phase diagram of the model, particularly focussing on changes of order parameters with the model parameters. Finally, in Section 4, we summarize the results of the present work.

\section{Model and Methods}

In this paper, we study a one-dimensional chain with a BCStype superconducting pairing term (i.e., $s$-wave one). The system is described by the attractive Hubbard model $(U<$ 0 ) in a magnetic field [40], which in the real space can be written in the following form:

$$
\hat{\mathcal{H}}=\sum_{\langle i, j\rangle \sigma}\left(-t-(\mu+\sigma h) \delta_{i j}\right) \hat{c}_{i \sigma}^{\dagger} \hat{c}_{j \sigma}+U \sum_{i} \hat{n}_{i \uparrow} \hat{n}_{i \downarrow}
$$

where $\hat{c}_{\sigma}^{\dagger}\left(\hat{c}_{\sigma}\right)$ denotes an operator of creation (annihilation) of the electron with spin $\sigma \in\{\uparrow, \downarrow\}$ at site $i$ and $\hat{n}_{i \sigma}=$ $\hat{c}_{i \sigma}^{\dagger} \hat{c}_{i \sigma}$ is particle number operator. $t>0$ is the hopping between the nearest-neighbor sites, and $U<0$ is the on-site pairing interaction. $\mu$ is the chemical potential, which determines the average number of particles $n=(1 / N) \sum_{i \sigma}\left\langle\hat{n}_{i \sigma}\right\rangle$ in the system (filling) ( $N$ is the total number of sites in the lattice). Finally, $h$ is a Zeeman field, which can originate from an external magnetic field (in $g \mu_{B} / 2$ units) or from population imbalance in the context of the cold atomic Fermi gases. Moreover, we can introduce $\mu_{\sigma}=\mu+\sigma h$ as the effective chemical potential of atoms with (pseudo) spin $\sigma$. The second term of Hamiltonian (1) is decoupled within the mean-field approximation, which takes into account only superconducting averages:

$\hat{n}_{i \uparrow} \hat{n}_{i \downarrow}=\Delta_{i}^{*} \hat{c}_{i \downarrow} \hat{c}_{i \uparrow}+\Delta_{i} \hat{c}_{i \uparrow}^{\dagger} \hat{c}_{i \downarrow}^{\dagger}-\left|\Delta_{i}\right|^{2}$,

where $\Delta_{i}=\left\langle\hat{c}_{i \downarrow} \hat{c}_{i \uparrow}\right\rangle$ is the site-dependent on-site superconducting order parameter (SOP). Then, the mean-field Hamiltonian in the real space is written in the form:

$$
\begin{aligned}
\hat{\mathcal{H}}^{M F}= & \sum_{\langle i, j\rangle \sigma}\left(-t-(\mu+\sigma h) \delta_{i j}\right) \hat{c}_{i \sigma}^{\dagger} \hat{c}_{j \sigma} \\
& +U \sum_{i}\left(\Delta_{i}^{*} \hat{c}_{i \downarrow} \hat{c}_{i \uparrow}+H . c .\right)-U \sum_{i}\left|\Delta_{i}\right|^{2} .
\end{aligned}
$$

Without loss of generality, one can write down the SOP as follows: $\Delta_{i}=\Delta_{0} \exp \left(i \boldsymbol{Q} \cdot \boldsymbol{R}_{i}\right)$, where $\Delta_{0}>0$ is the spatially oscillating amplitude of the SOP and $\boldsymbol{Q}$ is the total momentum of the Cooper pairs. We assume that the lattice constant is equal to one, i.e., $a=1$. In a one-dimensional case considered here, $|\boldsymbol{Q}|=Q_{x}$, where $Q_{x}$ is the (absolute value of) coordinate (the only one) of the vector $\boldsymbol{Q}$ (with the largest allowed value $Q_{x}^{\max } \equiv \pi$ ). The procedure of numerical solving of the system and final equations for the grand canonical potential and order parameters are presented in Refs. [40, 41]. Notice that all found solutions correspond to minimal value of the grand canonical potential (with respect to $\Delta_{0}$ and $\boldsymbol{Q}$ ) at fixed model parameters [40,41]. Below, we just discuss the behavior of the quantities in the system for some exemplary value of the on-site attraction.

\section{Numerical Results and Discussion}

In this section, we consider the system with a value of the pairing interaction set as $U / t=-3$. The ground-state phase diagram of model (3) is presented in Fig. 1. In the absence of an external Zeeman field, the usual superconducting BCStype $s$-wave state (with $\Delta_{0} \neq 0$ and $Q_{x}=0$ ) is stable. As the magnetic field increases, superfluidity is destroyed due to paramagnetic effects or by population imbalance. Hence, the unpolarized BCS-like superconducting phase undergoes a first-order phase transition to the polarized normal (NO) state (with $\Delta_{0}=0, Q_{x}$ - undetermined, and $|n-1| \neq 1$ ) or to the FFLO phase (with $\Delta_{0} \neq 0$ and $Q_{x} \neq 0$ ). Inside the FFLO phase, for large fields and near half-filling, the

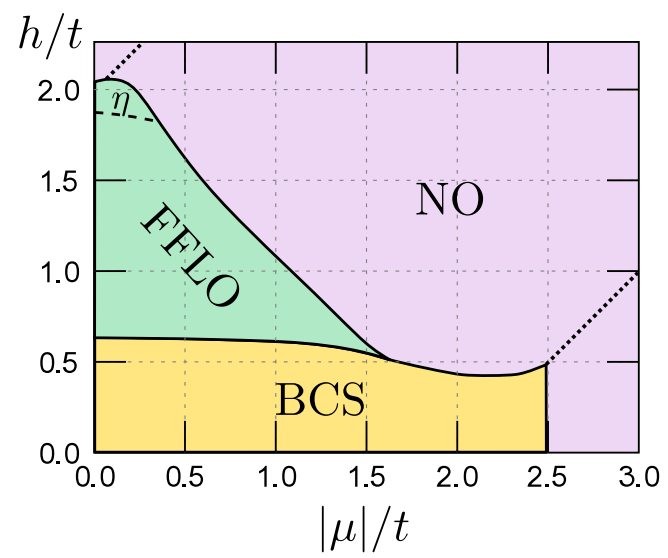

Fig. 1 The phase diagram of the model for $U / t=-3.0$ as a function of $|\mu| / t$ and $h / t$. BCS denotes the usual superconducting BCS-type $s$ wave state, FFLO labels the polarized superconducting state with nonzero momentum of Cooper pairs, whereas NO corresponds to normal (non-ordered) phase. Additionally, inside the FFLO region above the dashed line, there is a region, where the $\eta$ phase is distinguished. Inside the NO area, the NO phase with $n=1$ (for small $|\mu| / t$ and large $h / t$ ) and the NO phase with $|n-1|=1$ (for large $|\mu| / t$ and small $h / t$ ) are indicated by the dotted lines 
$\eta$-FFLO superconducting phase (i.e., the FFLO phase with $Q_{x}=Q_{x}^{\max }$ ) is also identified. Increasing higher the field and close to half-filling, the FFLO- $\eta$-pairing superconducting phase undergoes a first-order phase transition to the normal state (this transition can be also second-order for larger $|\mu| / t$, see the text below). Moreover, for magnetic fields larger than the top of the band (i.e., $2 t$ for onedimensional case studied here), one can find the magnetic Lifshitz transition [40, 42]. As a consequence of the relatively strong pairing interaction, the superconductivity still exists in the system, even if the Fermi surface for one type spin disappears.

Now, we discuss the evolution of (i) the amplitude $\Delta_{0}$ of the superconducting order parameter, (ii) the component $Q_{x}$ of the total momentum of Cooper pairs, and (iii) electron concentration $n$ in the system as a function of the model parameters $\mu$ and $h$.

Figure 2 presents the amplitude $\Delta_{0}$ of the superconducting order parameter. In the BCS phase, $\Delta_{0}$ is monotonously decreasing function of $|\mu| / t$ and it is independent of $h / t$. At the transition between the BCS phase and the NO phase with $n=0$, the $\Delta_{0}$ vanishes continuously as it should behave at continuous transition. At $h / t \neq 0$, the BCSNO transition is discontinuous (with discontinuous change of $\Delta_{0}$ ). Similarly, at the BCS-FFLO boundary, $\Delta_{0}$ exhibits a discontinuous change to a lower value. Inside the FFLO region (including also $\eta$ phase), $\Delta_{0}$ decreases monotonously with increasing $|\mu| / t$ and $h / t$. At the FFLO-NO boundary, parameter $\Delta_{0}$ vanishes continuously to $\Delta_{0}=0$ The transition between the $\eta$-FFLO phase and the NO phase is continuous only in some range of model parameters. For high magnetic field and near the half-filling, it is associated to a discontinuity of $\Delta_{0}$ (it is hardly visible in Fig. 2).

The ground-state values of $Q_{x}$ are presented in Fig. 3. As one can expect, $Q_{x}=0$ at the BCS phase, where the total momentum of Copper pairs is zero. In the FFLO phase, $Q_{x} \neq 0$ and it increases with increasing of the magnetic field to its maximal value $Q_{x}^{\max }=\pi$. In the whole

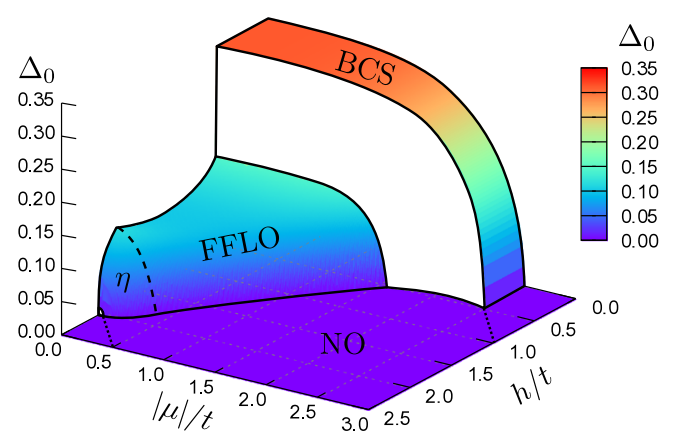

Fig. 2 The amplitude $\Delta_{0}$ of the superconducting order parameter for $U / t=-3.0$ as a function of $|\mu| / t$ and $h / t$. The white planes denote discontinuous changes of $\Delta_{0}$ at the boundaries between phases (notice the small region at the $\eta$-FFLO-NO boundary near $|\mu| / t=0$ )

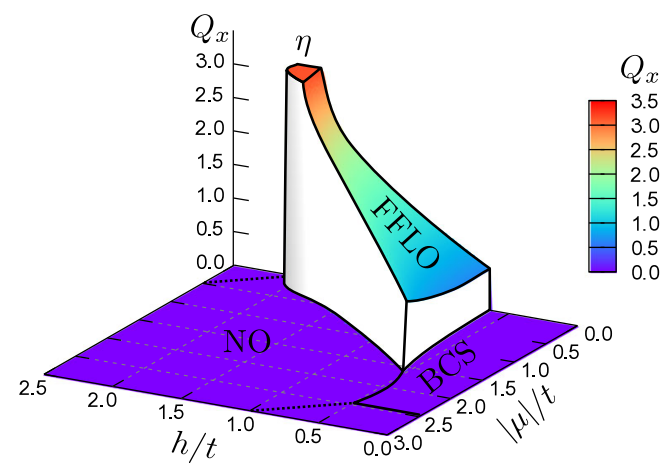

Fig. 3 The momentum $Q_{x}$ of superconducting pairs for $U / t=-3.0$ as a function of $|\mu| / t$ and $h / t$. At the $\eta$-FFLO phase, $Q_{x}=\pi$. In the NO region, $Q_{x}$ is undetermined (not well defined), but in the figure, we have adopted the convention that in the NO phase, $Q_{x}=0$. The white planes denote discontinuous changes of $Q_{x}$ at the boundaries between phases

region of $\eta$-FFLO phase, the total momentum of the pairs does not change and equals $Q_{x}=Q_{x}^{\max }$. Notice that at the BCS-FFLO boundary $Q_{x}$ changes discontinuously. $Q_{x}$ is continuous at the boundary between the $\eta$-FFLO phase (with $Q_{x}=Q_{x}^{\max }$ ) and the FFLO phase (where $0<Q_{x}<$ $Q_{x}^{\max }$ ). At the NO region, $Q_{x}$ is undetermined (i.e., it is not well defined, no Cooper pairs in the system), but in Fig. 3, we have adopted the convention that in the NO phase $Q_{x}=0$.

The very important feature is the behavior of the particle concentration $n$, particularly in the context of the phase separations (cf. Refs. [43-46] and references therein). If $n$ changes discontinuously from $n_{-}$to $n_{+}$at the boundary line between two phases in the diagram for fixed $\mu$, the phases can co-exist on the phase diagram as a function of $n$. The dependence of particle concentration $n$ (precisely the value of $|n-1|)$ with changing the model parameters is shown in Fig. 4. At fixed $h / t$, the value of $|n-1|$ is increasing function of $|\mu| / t$. It turns out that the discontinuous changes of $n$ occur at the same phase boundaries where $\Delta_{0}$ exhibits discontinuity. Namely, the BCS-FFLO, BCS-NO (only to the NO phase with $n \neq 0$ ) and $\eta$-FFLO-NO (only for high magnetic field and near the half-filling) transitions are associated to abrupt changes of $n$. The value of $|n-1|$ in the FFLO phase is smaller than those in the NO phase (at the FFLO-NO boundary). Similarly, at the BCS-FFLO boundary, the value of $|n-1|$ changes discontinuously to higher value in the FFLO phase. At the discontinuous BCS-NO boundary the relative values of concentrations depend on the value of $\mu / t$. For the discontinuous $\eta$-FFLO-NO transition, the lower value of $|n-1|$ is in the NO phase. As a result, one can distinguish three different phase-separated states, which can occur in the phase diagram as a function of $n$ : phase separation between the BCS and FFLO phases, phase separation between the BCS and NO phases, and finally phase separation between the $\eta$-FFLO and NO phases (cf. also [40]). 


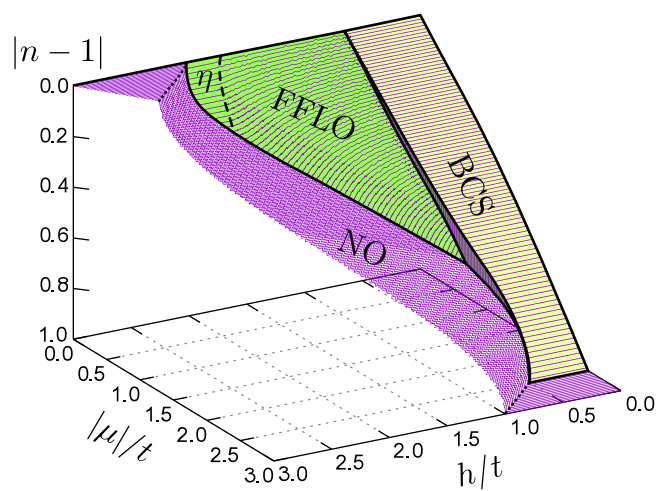

Fig. 4 The average concentration $|n-1|$ of the particles for fixed $U / t=-3.0$ as a function of $|\mu| / t$ and $h / t$. The discontinuities of $|n-1|$ occur at the same phase boundaries as those for $\Delta_{0}$, but they are less visible in the figure. Notice that $z$-axis of the plot (correponding to $|n-1|$ quantity) has inverted direction

The behavior of $\Delta_{0}, Q_{x}$, and $n$ should not strongly depend on low and intermediate values of $U<0$ qualitatively since it only determines the magnitude of the pairing potential in the system. Notice also that the ground-state phase diagrams for different attractive $U$ presented in Ref. [40] do not modify qualitatively with changing $U$ in this range of $U$.

\section{Summary}

In this work, we have studied the ground state of the attractive Hubbard model focusing on the behavior of order parameters. We found that in the ground state of the system the following phases can occur: the NO phase, the BCS phase, and the FFLO phase (with its extreme casethe $\eta$-FFLO phase). The phase transition between the BCS and FFLO phase is discontinuous one, whereas the FFLONO and FFLO $-\eta$-FFLO transitions are continuous. The BCS-NO and $\eta$-FFLO-NO transitions can be of both types, depending on the region of the phase diagram.

Notice that the mean-field approximation is generally valid only for small $U$ and high-dimensions. It overestimates usually critical temperatures and the range of stability of the phases with long-range order. However, the meanfield approach gives at least qualitative description of the system in the ground state, even in the strong coupling limit [4]. Nevertheless, we used this approach to study quasione-dimensional model describing fermionic ultracold gas on the optical lattice. According to the Mermin-Wagner theorem, a one-dimensional superfluid system cannot support superfluidity and would possess, at best, algebraically decaying long-range order at zero temperature [47] (cf. also with Refs. [48-50]). The real systems of atoms on the optical lattices are quasi-one-dimensional systems which means that the one-dimensional cylindrical-shaped regions are weakly coupled with each other, what makes the mean-field approximation more appropriate.

Acknowledgements The authors thank Jan Barański, Krzysztof Cichy, Anna Ciechan, Tadeusz Domański, and Matteo Rizzi for valuable comments and discussions.

Funding Information This work was partially supported by the National Science Centre (NCN, Poland) under grant nos. UMO-2016/20/S/ ST3/00274 (A.P.), UMO-2017/24/C/ST3/00357 (A.C.), UMO-2016/21/ D/ST3/03385 (K.J.K.), and UMO-2017/24/C/ST3/00276 (K.J.K.). K.R. acknowledges the support from CIBioFi and the Colombian Science, Technology and Innovation Foundation-COLCIENCIAS "Francisco José de Caldas" under project 1106-712-49884 (contract no. 264-2016) and -General Royalties System (Fondo CTeI-SGR) under contract no. BPIN 2013000100007.

Open Access This article is distributed under the terms of the Creative Commons Attribution 4.0 International License (http:// creativecommons.org/licenses/by/4.0/), which permits unrestricted use, distribution, and reproduction in any medium, provided you give appropriate credit to the original author(s) and the source, provide a link to the Creative Commons license, and indicate if changes were made.

\section{References}

1. Robaszkiewicz, S., Micnas, R., Chao, K.A.: Hartree theory for the negative- $U$ extended Hubbard model: ground state. Phys. Rev. B 24, 4018 (1981). https://doi.org/10.1103/PhysRevB.24.4018

2. Robaszkiewicz, S., Micnas, R., Chao, K.A.: Hartree theory for the negative- $U$, extended Hubbard model. II. Finite temperature. Phys. Rev. B 26, 3915 (1982). https://doi.org/10.1103/PhysRevB. 26.3915

3. Dutta, O., Gajda, M., Hauke, P., Lewenstein, M., Lühmann, D.S., Malomed, B.A., Sowiński, T., Zakrzewski, J.: Non-standard Hubbard models in optical lattices: a review. Rep. Prog. Phys. 78, 066001 (2015). https://doi.org/10.1088/0034-4885/78/6/066001

4. Micnas, R., Ranninger, J., Robaszkiewicz, S.: Superconductivity in narrow-band systems with local nonretarded attractive interactions. Rev. Mod. Phys. 62, 113 (1990). https://doi.org/10.1103/ RevModPhys.62.113

5. Bourdel, T., Khaykovich, L., Cubizolles, J., Zhang, J., Chevy, F., Teichmann, M., Tarruell, L., Kokkelmans, S.J.J.M.F., Salomon, C.: Experimental study of the BEC-BCS crossover region in lithium 6. Phys. Rev. Lett. 93, 050401 (2004). https://doi.org/10. 1103/PhysRevLett.93.050401

6. Cichy, A., Micnas, R.: The spin-imbalanced attractive Hubbard model in: phase diagrams and BCS-BEC crossover at low filling. Ann. Phys. 347, 207 (2014). https://doi.org/10.1016/j.aop.2014. 04.014

7. Fulde, P., Ferrell, R.A.: Superconductivity in a strong spinexchange field. Phys. Rev. 135, A550 (1964). https://doi.org/10.1103 /PhysRev.135.A550

8. Larkin, A.I., Ovchinnikov, Yu.N.: Nonuniform state of superconductors. Zh. Eksp. Teor. Fiz. 47, 1136 (1964). [Sov. Phys. JETP 20, 762 (1965)]

9. Jakubczyk, P.: Renormalization theory for the Fulde-FerrellLarkin-Ovchinnikov states at $T>0$. Phys. Rev. A 95, 063626 (2017). https://doi.org/10.1103/PhysRevA.95.063626 
10. Zocco, D.A., Grube, K., Eilers, F., Wolf, T., von Löhneysen, H.: Pauli-limited multiband superconductivity in $\mathrm{KFe}_{2} \mathrm{As}_{2}$. Phys. Rev. Lett. 111, 057007 (2013). https://doi.org/10.1103/PhysRevLett. 111.057007

11. Ptok, A., Crivelli, D.: The Fulde-Ferrell-Larkin-Ovchinnikov state in pnictides. J. Low Temp. Phys. 172, 226 (2013). https://doi.org/10.1007/s10909-013-0871-0

12. Kasahara, S., Watashige, T., Hanaguri, T., Kohsaka, Y., Yamashita, T., Shimoyama, Y., Mizukami, Y., Endo, R., Ikeda, H., Aoyama, K., Terashima, T., Taichi, S., Wolf, T., von Löhneysen, H., Shibauchi, T., Matsuda, Y.: Field-induced superconducting phase of FeSe in the BCS-BEC cross-over. PNAS 111, 16309 (2014). https://doi.org/10.1073/pnas.1413477111

13. Cho, Ch.-w., Yang, J.H., Shen, J., Wolf, T., Lortz, R.: Thermodynamic evidence for the Fulde-Ferrell-Larkin-Ovchinnikov state in the $\mathrm{KFe}_{2} \mathrm{As}_{2}$ superconductor. arXiv:1708.05526

14. Ptok, A., Kapcia, K.J., Piekarz, P., Oleś, A.M.: The ab initio study of unconventional superconductivity in $\mathrm{CeCoIn}_{5}$ and FeSe. New. J. Phys. 19, 063039 (2017). https://doi.org/10.1088/1367-2630/ aa6d9d

15. Bianchi, A., Movshovich, R., Oeschler, N., Gegenwart, P., Steglich, F., Thompson, J.D., Pagliuso, P.G., Sarrao, J.L.: Firstorder superconducting phase transition in $\mathrm{CeCoIn}_{5}$. Phys. Rev. Lett. 89, 137002 (2002). https://doi.org/10.1103/PhysRevLett.89. 137002

16. Bianchi, A., Movshovich, R., Capan, C., Pagliuso, P.G., Sarrao, J.L.: Possible Fulde-Ferrell-Larkin-Ovchinnikov superconducting state in CeCoIn 5 . Phys. Rev. Lett. 91, 187004 (2003). https://doi.org/10.1103/PhysRevLett.91.187004

17. Matsuda, Y., Shimahara, H.: Fulde-Ferrell-Larkin-Ovchinnikov state in heavy fermion superconductors. J. Phys. Soc. Jpn. 76, 051005 (2007). https://doi.org/10.1143/JPSJ.76.051005

18. Kenzelmann, M., Strässle, Th., Niedermayer, Ch., Sigrist, M., Padmanabhan, B., Zolliker, M., Bianchi, A.D., Movshovich, R., Bauer, E.D., Sarrao, J.L., Thompson, J.D.: Coupled superconducting and magnetic order in CeCoIn 5 . Science 321, 1652 (2008). https://doi.org/10.1126/science.1161818

19. Maśka, M.M., Mierzejewski, M., Kaczmarczyk, J., Spałek, J.: Superconducting Bardeen-Cooper-Schrieffer versus FuldeFerrell-Larkin-Ovchinnikov states of heavy quasiparticles with spin-dependent masses and Kondo-type pairing. Phys. Rev. B 82, 054509 (2010). https://doi.org/10.1103/PhysRevB.82.054509

20. Kaczmarczyk, J., Spałek, J.: Unconventional superconducting phases in a correlated two-dimensional Fermi gas of nonstandard quasiparticles: a simple model. J. Phys.: Condens. Matter 22, 355702 (2010). https://doi.org/10.1088/0953-8984/22/35/355702

21. Uji, S., Terashima, T., Nishimura, M., Takahide, Y., Konoike, T., Enomoto, K., Cui, H., Kobayashi, H., Kobayashi, A., Tanaka, H., Tokumoto, M., Choi, E.S., Tokumoto, T., Graf, D., Brooks, J.S.: Vortex dynamics and the Fulde-Ferrell-Larkin-Ovchinnikov state in a magnetic-field-induced organic superconductor. Phys. Rev. Lett. 97, 157001 (2006). https://doi.org/10.1103/PhysRevLett.97. 157001

22. Lortz, R., Wang, Y., Demuer, A., Böttger, P.H.M., Bergk, B., Zwicknagl, G., Nakazawa, Y., Wosnitza, J.: Calorimetric evidence for a Fulde-Ferrell-Larkin-Ovchinnikov superconducting state in the layered organic superconductor $\kappa$ (BECT-TTF $)_{2} \mathrm{Cu}(\mathrm{NCS})_{2}$. Phys. Rev. Lett. 99, 187002 (2007). https://doi.org/10.1103/PhysRevLett.99.187002

23. Mayaffre, H., Kramer, S., Horvatic, M., Berthier, C., Miyagawa, K., Kanoda, K., Mitrovic, V.F.: Evidence of Andreev bound states as a hallmark of the FFLO phase in $\kappa$-(BEDT-TTF $)_{2} \mathrm{Cu}(\mathrm{NCS})_{2}$. Nat. Phys. 10, 928 (2014). https://doi.org/10.1038/nphys3121

24. Liu, W.V., Wilczek, F.: Interior gap superfluidity. Phys. Rev. Lett. 90, 047002 (2003). https://doi.org/10.1103/PhysRevLett.90.047 002
25. Hu, H., Liu, X.-J.: Mean-field phase diagrams of imbalanced Fermi gases near a Feshbach resonance. Phys. Rev. A 73, 051603 (2006). https://doi.org/10.1103/PhysRevA.73.051603

26. Guan, X.W., Batchelor, M.T., Lee, C., Bortz, M.: Phase transitions and pairing signature in strongly attractive Fermi atomic gases. Phys. Rev. B 76, 085120 (2007). https://doi.org/10.1103/PhysRev B.76.085120

27. Orso, G.: Attractive fermi gases with unequal spin populations in highly elongated traps. Phys. Rev. Lett. 98, 070402 (2007). https://doi.org/10.1103/PhysRevLett.98.070402

28. Hu, H., Liu, X.-J., Drummond, P.D.: Phase diagram of a strongly interacting polarized Fermi gas in one dimension. Phys. Rev. Lett. 98, 070403 (2007). https://doi.org/10.1103/PhysRevLett.98.070 403

29. Lüscher, A., Noack, R.M., Läuchli, A.M.: Fulde-Ferrell-LarkinOvchinnikov state in the one-dimensional attractive Hubbard model and its fingerprint in spatial noise correlations. Phys. Rev. A 78, 013637 (2008). https://doi.org/10.1103/PhysRevA.78.013637

30. Pahl, S., Koinov, Z.: Phase diagram of a ${ }^{6} \mathrm{Li}^{-}{ }^{40} \mathrm{~K}$ mixture in a square lattice. J. Low Temp. Phys. 176, 113 (2014). http://doi. org/10.1007/s10909-014-1166-9

31. Wolak, M.J., Grémaud, B., Scalettar, R.T., Batrouni, G.G.: Pairing in a two-dimensional Fermi gas with population imbalance. Phys. Rev. A 86, 023630 (2012). https://doi.org/10.1103/PhysRevA.86. 023630

32. Guan, X.-W., Batchelor, M.T., Lee, Ch.: Fermi gases in one dimension: from Bethe ansatz to experiments. Rev. Mod. Phys. 85, 1633 (2013). https://doi.org/10.1103/RevModPhys.85.1633

33. Cichy, A., Cichy, K., Polak, T.P.: Competition between Abelian and Zeeman magnetic field effects in a two-dimensional ultracold gas of fermions. Ann. Phys. 354, 89 (2015). https://doi.org/10.10 16/j.aop.2014.12.008

34. Hu, A., Maśka, M.M., Clark, Ch.W., Freericks, J.K.: Robust finitetemperature disordered Mott-insulating phases in inhomogeneous Fermi-Fermi mixtures with density and mass imbalance. Phys. Rev. A 91, 063624 (2015). https://doi.org/10.1103/PhysRevA.91. 063624

35. Fedichev, P.O., Kagan, Yu., Shlyapnikov, G.V., Walraven, J.T.M.: Influence of nearly resonant light on the scattering length in low-temperature atomic gases. Phys. Rev. Lett. 77, 2913 (1996). https://doi.org/10.1103/PhysRevLett.77.2913

36. Partridge, G.B., Li, W., Kamar, R.I., Liao, Y.-A., Hulet, R.G.: Pairing and phase separation in a polarized Fermi gas. Science 311, 503 (2006). https://doi.org/10.1126/science.1122876

37. Zwierlein, M.W., Ketterle, W.: Comment on "Pairing and phase separation in a polarized Fermi gas". Science 314, 54 (2006). https://doi.org/10.1126/science.1129812

38. Schunck, C.H., Shin, Y., Schirotzek, A., Zwierlein, M.W., Ketterle, W.: Pairing without superfluidity: the ground state of an imbalanced Fermi mixture. Science 316, 867 (2007). https://doi.org/10.1126/science.1140749

39. Georgescu, I.M., Ashhab, S., Nori, F.: Quantum simulation. Rev. Mod. Phys. 86, 153 (2014). https://doi.org/10.1103/RevModPhys. 86.153

40. Ptok, A., Cichy, A., Rodríguez, K., Kapcia, K.J.: Critical behavior in one dimension: unconventional pairing, phase separation, BECBCS crossover, and magnetic Lifshitz transition. Phys. Rev. A 95, 033613 (2017). https://doi.org/10.1103/PhysRevA.95.033613

41. Januszewski, M., Ptok, A., Crivelli, D., Gardas, B.: GPU-based acceleration of free energy calculations in solid state physics. Comput. Phys. Commun. 192, 220 (2015). https://doi.org/10.1016 /j.cpc.2015.02.012

42. Ptok, A., Kapcia, K.J., Cichy, A., Oleś, A.M., Piekarz, P.: Magnetic Lifshitz transition and its consequences in multi-band ironbased superconductors. Sci. Rep. 7, 41979 (2017). https://doi.org/ 10.1038/srep41979 
43. Arrigoni, E., Strinati, G.C.: Doping-induced incommensurate antiferromagnetism in a Mott-Hubbard insulator. Phys. Rev. B 44, 7455 (1991). https://doi.org/10.1103/PhysRevB.44.7455

44. Bąk, M.: Mixed phase and bound states in the phase diagram of the extended Hubbard model. Acta Phys. Pol. A 106, 637 (2004). https://doi.org/10.12693/APhysPolA.106.637

45. Kapcia, K., Robaszkiewicz, S.: The magnetic field induced phase separation in a model of a superconductor with local electron pairing. J. Phys.: Condens. Matter 25, 065603 (2013). https://doi.org/10.1088/0953-8984/25/6/065603

46. Kapcia, K.J., Czart, W.R., Ptok, A.: Phase separation of superconducting phases in the Penson-Kolb-Hubbard model. J. Phys. Soc. Jpn. 85, 044708 (2016). https://doi.org/10.7566/JPSJ.85.044708
47. Mermin, N.D., Wagner, H.: Absence of ferromagnetism or antiferromagnetism in one- or two-dimensional isotropic Heisenberg models. Phys. Rev. Lett. 17, 1133 (1966). https://doi.org/10.1103/ PhysRevLett.17.1133

48. Lieb, E.H.: Two theorems on the Hubbard model. Phys. Rev. Lett. 62, 1201 (1989). https://doi.org/10.1103/PhysRevLett.62.1201

49. Uhrig, G.S.: Nonexistence of planar magnetic order in the oneand two-dimensional generalized Hubbard model at finite temperatures. Phys. Rev. B 45, 4738 (1989). https://doi.org/10.1103/Phys RevB.45.4738

50. Müller-Hartmann, E.: Correlated fermions on a lattice in high dimensions. Z. Phys. B 74, 507 (1989). https://doi.org/10.1007/ BF01311 